\title{
Efficacy of tazarotene cream plus clindamycin gel vs tretinoin cream plus clindamycin gel in treatment of mild to moderate acne vulgaris: a randomized trial
}

Paudel $S^{1}$, Agrawal $S^{2}$, Dhali $T K^{3}$

${ }^{I}$ Registrar, Civil Service Hospital, Kathmandu, Nepal ${ }^{2}$ Professor, Department of Dermatology \& Venereology, B. P. Koirala Institute of Health Sciences, Dharan, Nepal. ${ }^{3}$ Associate Professor, ESIC Hospital, Delhi, India

Funding: None

Conflicts of interest: None

\begin{abstract}
Introduction: Since the cause of acne is multi-factorial, currently the combination of a topical retinoid and an antibiotic is recommended for the treatment of acne.

Objectives: To compare the efficacy and tolerability of tazarotene $0.1 \%$ cream plus clindamycin $1 \%$ gel (Taz + Clin) with tretinoin $0.025 \%$ cream plus clindamycin $1 \%$ gel (Tret + Clin) in treatment of mild to moderate acne vulgaris and also to evaluate the quality of life in these patients.

Materials and Methods: Patients 12 years of age and older with mild to moderate acne vulgaris (N $=146$ ) treated either with Tazarotene or tretinoin in the evening and clindamycin in the morning for 12 weeks participated in the assessor blinded randomized parallel group study. The Global Acne Grading System (GAGS) score, inflammatory, non-inflammatory and total lesion count change and local side effects were assessed at 4, 8 and 12 weeks follow up.
\end{abstract}

Results: Treatment with Taz + Clin resulted in a significantly superior reduction compared to Tret + Clin at 12 weeks in median GAGS score $(57.14 \%$ Vs $37.52 \%)$, non-inflammatory lesions $(86.02 \% \mathrm{Vs}$, $80.4 \%$ ), inflammatory lesions (100\% Vs $94.03 \%)$ and total lesions count (87.47\% Vs $81.08 \%)$. Both the regimens were well tolerated. There was a significant improvement in the patients' quality of life by a significant reduction of Dermatology Life Quality Index (DLQI) score from baseline to week 12 in Taz+Clin group (81.2\%) and Tret+Clin group (66.6\%).

Conclusion: Tazarotene plus clindamycin offers significantly greater efficacy and well tolerability to tretinoin plus clindamycin regimen in the treatment of mild to moderate acne.

Keywords: Tazarotene, Tretinoin, Clindamycin, DLQI, Acne, Nepal

Address for correspondence:

Dr. Sudha Agrawal

Professor \& Head

Department of Dermatology and Venereology

B. P. Koirala Institute of Health Sciences, Dharan, Nepal.

Fax: 00977-25-520251

E-mail: sudha92@yahoo.com 


\section{Original Article}

\section{Introduction}

Acne vulgaris is a chronic inflammatory disease of the pilosebaceous units, affecting approximately $80 \%$ of young adults and adolescents. ${ }^{1}$ If not appropriately treated, acne may cause serious physical and emotional scarring. It can significantly affect the quality of life. ${ }^{2}$ The cornerstone of therapy in case of mild to moderate acne is the topical retinoids as they inhibit the development of micro comedones, the precursor of all other acne lesions. ${ }^{3}$ Topical retinoids have been used in acne therapy since 1962 the first one being Tretinoin. Only recently, within last 10 years, the receptor specific retinoids are extensively being introduced in the treatment of acne with improved efficacy and tolerability, the most recent one being tazarotene. ${ }^{4,5}$ In a recent consensus conference, the combination of a topical retinoid and an antibiotic is recommended for the treatment of acne in a majority of patients with mild to moderate disease. ${ }^{2,6}$

Data from several randomized, double-blind, comparative studies indicate that tazarotene $0.1 \%$ gel and cream may be more effective than tretinoin gel or cream in the treatment of mild to moderate facial acne vulgaris. ${ }^{7-9}$ Therefore it is likely that tazarotene plus clindamycin may offer superior efficacy to tretinoin plus clindamycin. A study has been conducted in past with this hypothesis ${ }^{3}$ and we have also endeavored to undertake study with same hypothesis. In this study we compared the efficacy and tolerability of Tazarotene $0.1 \%$ cream plus Clindamycin 1\% gel with Tretinoin $0.025 \%$ cream plus Clindamycin $1 \%$ gel in the treatment of mild to moderate acne vulgaris and also evaluated the quality of life before and after the treatment.

\section{Material and Methods Patients:}

Patients who were 12 years and older with mild to moderate facial and or truncal acne vulgaris on Global Acne Grading System (GAGS) ${ }^{10}$ score were eligible for enrolment. A total of 146 acne patients (73 in each group) were enrolled in this study to detect clinically significant difference between-group of $10 \%$ in the total lesion count and GAGS score at 12 weeks. Following statistical parameters were assumed to be true in this study; a standard deviation of $20 \%$ in groups, a two sided test, an alpha of 0.05 , a power of 0.8 and dropout rate of $15 \%$.

Exclusion criteria included any nodulocystic lesions at baseline, acne global score more than 30 in GAGS, pregnancy or breastfeeding, use of topical antibiotics or any acne medication in past 14 days, use of systemic antibiotics in past 30 days, use of Oral Contraceptive Pills in past 3 months, use of oral retinoids in past 12 months, history of intolerance or hypersensitivity by any drug under study, any skin disorder that might interfere with the diagnosis or evaluation of acne vulgaris, history of facial procedure (e.g. facial, chemical or laser peel, micro-dermabrasion) 2 weeks before study.

This was an assessor blind randomized parallel group study. A computer generated randomization list was generated to produce two parallel groups (1:1 ratio) of patients, 73 in each group. Each patient was assigned a number at enrolment which defined a study drug assignment. In tazarotene group, patients applied one-fourth fingertip unit $(0.112 \mathrm{gm})$ of tazarotene $0.1 \%$ cream (Tazret $^{\mathrm{TM}}$ $0.1 \%$ cream) over the face and additional onefourth fingertip unit to back/chest (if affected), in the evening. In the morning the patients were to wash and dry the affected area and apply one finger tip unit $(0.45 \mathrm{gm})$ clindamycin $1 \%$ gel (Clingard $^{\mathrm{TM}} 1 \%$ gel) over the affected area. Similarly in tretinoin group applied same amount $(0.112 \mathrm{gm})$ of tretinoin $0.025 \%$ cream (Trewor $^{\mathrm{TM}}$ $0.025 \%$ cream and Retino- $\mathrm{A}^{\mathrm{TM}} 0.025 \%$ cream) in the similar way followed in the morning by the same dose of clindamycin 1\% gel (Clingard ${ }^{\mathrm{TM}}$ $1 \%$ gel). No other medication or cosmetics was allowed to use during the study period. Patients were requested to avoid excessive sun exposure. All treatments were administered from baseline through 12 weeks. Evaluation was done at baseline, 4,8 and 12 weeks.

This study was approved by the local scientific research committee. All patients (and guardians 


\section{Original Article}

if necessary) were required to give written consent before enrolment.

\section{Assessments:}

For efficacy evaluation the investigator evaluated the patients at 4 weeks interval in terms of GAGS score, non-inflammatory, inflammatory and total lesion count. Macules were not included in the lesion count. The efficacy analysis was conducted as per protocol and the intent to treat population. The primary efficacy end points were calculated on the basis of the percent reduction from baseline to week 12 in the GAGS scores and lesion counts. The response rates (percentage of patients achieving 50\% reduction in the total GAGS score, non-inflammatory, inflammatory and total lesions) was also compared at 12 weeks in each treatment group. For all patients who discontinued treatment before 12 weeks the last observation was carried forward for all efficacy end points for Intention to treat analysis.

For the tolerability evaluation dryness, pruritus and burning sensation were reported by patients themselves on a scale of 0 (none), 1 (mild), 2 (moderate) and 3 (severe), while erythema and scaling were assessed by investigator. Any tolerance parameter classified as 'severe' was recorded as an adverse event.

\section{Quality of life:}

Each patient was asked to fill the questionnaire intended to measure quality of life using the standardized Nepali version of Dermatology Life Quality Index (DLQI). ${ }^{13}$ They were asked to respond the same set of questionnaire at 12 weeks also. The median percentage change/reduction in DLQI score from the baseline to 12 weeks and in between the groups was considered as the secondary efficacy end-point.

\section{Statistical analysis:}

Statistical analysis was conducted both perprotocol and intention-to treat population (defined as all enrolled patients to whom study drug was dispensed; with the last observation carried forward) basis using two sided tests. An alpha of 0.05 was considered significant. All data was evaluated on the software SPSS version 13 for the comparison of the two groups at the base line and between group differences in the percentage reduction of lesion counts or GAGS scores by using the Wilcoxon rank sum test. The mean score of dryness, erythema, scaling, stinging/burning and pruritus were also evaluated using Wilcoxon rank sum test. A Chi square test was used to evaluate the categorical variables including response rates (the between group difference in the incidence of more than or equal to $50 \%$ improvement in GAGS scores, inflammatory, non-inflammatory and total lesion counts). Non-parametric statistics were used (Wilcoxon matched pairs and Wilcoxon rank sum test) to assess the quality of life before and after the treatment and between the treatment groups.

\section{Results}

\section{Baseline characteristics:}

Of total 146 patients (73 in each group), one hundred and thirty four patients $(91.8 \%)$ completed the study, with the main reasons for discontinuation being noncompliance and unavailability for follow up (figure1). The two groups were well matched at the baseline variables (table 1).

Among 146 patients of mild to moderate acne vulgaris $66(45 \%)$ were males and $80(55 \%)$ were females with equal distribution in both the groups and with male to female ratio of 1:1.2. The age of the patients ranged from 13 to 32 years with the mean of $19.14 \pm 3.15$ years. The maximum number of cases, $85(58.21 \%)$ were in the less than 20 years age group. Majority of the patients seeking treatment were the students of grade 11 and $12,45 \%$, in both groups. Patients presented to us for the treatment with the duration ranging from 7 days to 7 years. Sixty (41.1\%) patients presented within one year of the disease onset. Among the female patients 46 (57.5\%) reported the flare up of the acne lesions during the menses, and $34(42.5 \%)$ reported no effect. Females who had flaring of disease during menstruation had that before the onset of menstruation, that was, 44 (95.65\%). Fifty-one (34.93\%) patients reported that the sun exposure aggravates their acne. Forty- 


\section{Original Article}

one $(28 \%)$ patients said stress increases their acne lesions. Forty-eight (32.87\%) patients had family history of acne. Forty-five (30.82\%) patients had taken treatment for acne in the past. Most of the patients had lesion on the face followed by lesions over face, chest and back in both the groups. In tazarotene/clindamycin group, $49(67.12 \%)$ had lesions only on the face, $5(6.84 \%)$ on face and chest, $14(19.19 \%)$ on face, chest and back and $5(6.84 \%)$ on face and back. While in tretinoin/clindamycin group $53(72.6 \%)$ had only facial involvement, 2 (2.73\%) face and chest, 12 $(16.43 \%)$ face, chest and back and $6(8.21 \%)$ patients had lesions on face and back.

\section{Efficacy evaluation}

For both treatment groups, a progressive decline was observed in the GAGS score and lesion count. The percent reduction in GAGS score from the baseline was greater in tazarotene/cindamycin regimen in all three follow ups compared to tretinoin/clindamycin regimen but the difference reached the statistical significance only at week 12, (at week 4, $\mathrm{p}=0.178$; at week $8, \mathrm{p}=0.189$; and at week $12(\mathrm{p}<0.001)$ (figure 2$)$.

There was greater reduction in non-inflammatory lesion count in all three follow-ups in tazarotene/clindamycin group compared to tretinoin/ clindamycin group and the difference remained till the end and at 12 weeks the reduction in tazarotene/clindamycin regimen was $86.02 \%$ while that in tretinoin/clindamycin group reduction rate was $80.4 \%(\mathrm{p}=0.013)$. The reduction in the tazarotene/clindamycin regimen in all the followups reached the statistical significance (figure 3).

The reduction in the inflammatory lesions (papules and pustules) within the tazarotene/clindamycin and tretinoin/clindamycin regimens at all three follow-ups was significant but inter-group analysis of reduction in count was significant only at week 12 , which was $100 \%$ in the former and $94.03 \%$ in the latter $(\mathrm{p}=0.024)$ (figure 4$)$.

At the baseline the mean number of inflammatory and non-inflammatory lesions combined was $136.58 \pm 82.07$ with median of 121.00 in tazarotene/clindamycin group as compared to $137.11 \pm 118.25$ with median of 101.00 in tretinoin/clindamycin group. The percent reduction in median was greater in tazarotene/clindamycin group compared to tretinoin/clindamycin group in all three follow ups and at 12 weeks the median reduction in total lesion count in former group was $87.47 \%$ to that of $81.08 \%$ in the latter $(p=0.008)$ (figure 5).

\section{Efficacy evaluation as Intention to treat analysis}

The median percent reduction in GAGS score and the lesion count from baseline to week 12 was greater in tazarotene/clindamycin regimen than the tretinoin/clindamycin regimen with significant $\mathrm{p}$ value for all observations (Table 2). This observation was similar to that of per protocol analysis. The number of patients achieving at least 50\% reduction in GAGS score and lesion count was more in tazarotene/clindamycin group compared to tretinoin/clindamycin group and the difference was significant in all efficacy parameters (figure $6)$.

\section{Improvement in Dermatology Life Quality Index (DLQI) \\ Reduction in the DLQI score}

There was a significant improvement in the patients' quality of life by a significant reduction of DLQI score from baseline to week 12. The mean DLQI score in tazarotene/clindamycin group at the baseline was $8.89 \pm 7.04$ and the median was 8.00 . The mean score reduced to $2.70 \pm 3.36$ and the median to 1.50 after 12 weeks of treatment, a reduction of $81.25 \%$ from baseline median $(p<0.001)$. In the tretinoin/ clindamycin group the mean DLQI score at the baseline was $7.47 \pm 5.68$ with the median of 6.00 and at 12 weeks, $2.94 \pm 4.07$, and 2.00 ; a $66.66 \%$ reduction in median from the baseline $(<0.001)$ (Figure 7). The difference in reduction between the groups did not reach the level of significance $(\mathrm{p}=0.094)$.

\section{Tolerability and adverse event}

Five local skin tolerability variables (erythema, scaling, dryness, pruritus and stinging/burning sensations) were graded from 0 (none) to 3 


\section{Original Article}

(severe) in 4-points scale. In tazarotene/ clindamycin group 59(80.82\%) patients had at least one local sign/symptom developed during the entire follow-up period. Likewise 65(89.04\%) patients in tretinoin/ clindamycin group experienced at least one local sign/symptom during the follow-ups. None of the patients in both the groups experienced severe score (adverse event).

\section{Discussion:}

The combination therapy with topical tazarotene/ tretinoin and clindamycin gel was demonstrated to be a very effective treatment for patients with mild to moderate acne, and it was likely that clindamycin enhanced the clinical efficacy of tazarotene/ tretinoin. Furthermore, when the two study treatment regimens were compared, the tazarotene/clindamycin regimen appeared superior towards efficacy in the present study.

There was greater reduction in non-inflammatory lesion count in all three follow-ups in tazarotene/clindamycin group compared to tretinoin/clindamycin group; at 4 weeks $44.00 \%$ vs. $30.47 \%(\mathrm{p}=0.011)$, at 8 weeks $70.76 \%$ vs. $63.72 \%(\mathrm{p}=0.042)$ and at 12 weeks $86.02 \%$ vs. $80.4 \%(p=0.013)$. In a similar study in past ${ }^{3}$ tazarotene/clindamycin achieved greater reductions than tretinoin/clindamycin in noninflammatory lesion count $(71 \%$ vs. $52 \%$ at week $12, \mathrm{p}<0.05)$. The reduction in our study was greater than this study and we also got significant difference between two groups. Webster et al, $(2001)^{7}$ when compared tazarotene gel $0.1 \%$ with tretinoin $0.025 \%$ gel once daily for 12 weeks, found that tazarotene was more effective than tretinoin $0.025 \%$ gel in reducing the noninflammatory lesions $(\mathrm{p}<0.05)$. Leyden et al, $(2002)^{8}$ compared tazarotene $0.1 \%$ gel and tretinoin $0.1 \%$ microsponge gel, applied once daily for 12 weeks they also found more reduction in the non-inflammatory lesion in tazarotene group, $60 \%$ vs $38 \%, p=0.02$. In the latter two studies the reduction was lesser than in our study and it could be due to the use of clindamycin gel along with retinoids.
In the present study the reduction in the median inflammatory lesions from baseline to week 12 was more in tazarotene/clindamycin group compared to tretinoin/clindamycin group, $100.00 \%$ vs. $94.03 \%(p=0.024)$. Similarly Tanghetti et al $(2007)^{3}$ also found more reduction in tazarotene/clindamycin group compared to tretinoin/clindamycin group, $77 \%$ vs. $67 \%$ at week $12, p=0.053$. Superior response in our study could be due to exclusion of macules from count or better efficacy of tazarotene and clindamycin with separate application.

The patients achieving at least $50 \%$ reduction in GAGS score and lesion count was more in tazarotene/clindamycin group compared to tretinoin/clindamycin group at 12 weeks and the difference was significant for GAGS score, $64.4 \%$ vs. $28.8 \%(\mathrm{p}<0.001)$, non-inflammatory lesion count $91.8 \%$ vs. $79.5 \%(\mathrm{p}=0.034)$ and total lesion count $95.9 \%$ vs. $83.6 \%(p=0.029)$. This observation was similar to Tanghetti et al, $(2007)^{3}$ observation for $>50 \%$ global improvement. They found that greater percentage of patients achieved $>50 \%$ global improvement with tazarotene/ clindamycin than with tretinoin/clindamycin $88 \%$ vs. $75 \%(\mathrm{p}<0.05)$. Leyden et al, $(2002)^{8}$ also reported $>50 \%$ global improvement in significantly more number of patients in tazarotene/clindamycin group, $67 \%$ vs. $49 \%$, $\mathrm{p}=0.03$.

The mean scaling and burning scores were higher in tazarotene/clindamycin regimen compared to tretinoin/clindamycin regimen in the first followup reaching statistical significance and declined markedly in both groups over the course of treatment, suggesting that the clindamycin gel itself may have been of therapeutic value in reducing the irritant effects of retinoids. ${ }^{12}$ Tanghetti et al $(2007)^{3}$ also observed that these local side effects were minimal and well tolerated in their study population. Webster et al, (2001) ${ }^{7}$ observed slightly more irritation with tazarotene therapy compared to tretinoin $0.025 \%$ gel with all other side effects being trace and tolerable. Leyden et al, $(2002)^{8}$ also observed only trace mean level of side effects and no clinical significance in 


\section{Original Article}

group difference, however in their study two patients from each group discontinued the treatment owing to treatment related side effects.

Acne vulgaris significantly affects the quality of life and for many years, has been shown to be associated with psychological morbidity. ${ }^{13}$ The results of the present study revealed that acne affected the patients quality of life moderately and there was considerable improvement in the patients' quality of life by a significant reduction of DLQI score from baseline to week 12 in both the treatment groups. We chose DLQI over other instruments to measure quality of life because it is a self-explanatory, graduated scale, which includes most of the aspects of QOL variables, and can simply be handed to the patient.

Although there was no similar study done in the past comparing the QOL in tazarotene and tretinoin, Baz and colleagues $(2004)^{14}$ observed that the disability caused by acne can be largely reversed by effective treatment. In our study also the quality of life was improved accordingly with the objective clinical improvement from baseline to 12 weeks in both the treatment groups. The number of patients achieving the DLQI scores of zero following treatment was more in tazarotene/clindamycin group, $28(40 \%)$ than in tretinoin/clindamycin group 16 (25\%). This favors that DLQI was also most responsive to change as supported by clinical efficacy of tazarotene/clindamycin regimen.

\section{Conclusion:}

Tazarotene plus clindamycin offers significantly greater efficacy than tretinoin plus clindamycin when used once daily for the treatment of mild to moderate acne vulgaris. The local side effects are minimal and well tolerated in both the treatment groups which gradually reduce to almost null with subsequent use. The quality of life also becomes better after the use of these drugs. 


\section{Original Article}

Table 1. Baseline characterstics of the study population

\begin{tabular}{|c|c|c|c|c|c|}
\hline \multirow{2}{*}{\multicolumn{2}{|c|}{ Characteristics }} & \multicolumn{2}{|c|}{ Group } & \multirow[b]{2}{*}{ P value } & \multirow[b]{2}{*}{ Remarks } \\
\hline & & \multirow{2}{*}{$\begin{array}{c}\begin{array}{c}\text { Tazarotene/ } \\
\text { clindamycin } \\
(n=73)\end{array} \\
19.26 \pm 2.68\end{array}$} & $\begin{array}{c}\text { Tretinoin/ } \\
\text { clindamycin } \\
(\mathrm{n}=73)\end{array}$ & & \\
\hline \multirow[t]{2}{*}{ Age (years) } & Mean \pm SD & & $19.03 \pm 3.57$ & \multirow[t]{2}{*}{0.656} & \multirow[t]{2}{*}{ NS } \\
\hline & Median & 19.00 & 19.00 & & \\
\hline \multirow[t]{2}{*}{ Sex, No. (\%) } & Male & $33(45.20)$ & $33(45.20)$ & \multirow[t]{2}{*}{1.00} & \multirow[t]{2}{*}{ NS } \\
\hline & Female & $40(54.79)$ & $40(54.79)$ & & \\
\hline \multirow[t]{2}{*}{ Duration (mths) } & Mean \pm SD & $25.14 \pm 17.41$ & $23.14 \pm 19.26$ & \multirow[t]{2}{*}{0.510} & \multirow[t]{2}{*}{ NS } \\
\hline & Median & 24.00 & 24.00 & & \\
\hline \multirow{2}{*}{$\begin{array}{l}\text { Family History, } \\
\text { No. }(\%)\end{array}$} & Absent & $38(52.05)$ & $44(60.27)$ & \multirow[t]{2}{*}{0.230} & \multirow[t]{2}{*}{ NS } \\
\hline & Present & $35(47.94)$ & $29(39.72)$ & & \\
\hline \multicolumn{6}{|l|}{ Site of lesion } \\
\hline Site, No. (\%) & S1 & $49(67.12)$ & $53(72.60)$ & 0.630 & \multirow[t]{4}{*}{ NS } \\
\hline S1- Face, & $\mathrm{S} 1, \mathrm{~S} 2$ & $5(6.84)$ & $2(2.73)$ & & \\
\hline S2-Chest & $\mathrm{S} 1, \mathrm{~S} 2, \mathrm{~S} 3$ & $14(19.17)$ & $12(16.43)$ & & \\
\hline S3- Back & $\mathrm{S} 1, \mathrm{~S} 3$ & $5(6.84)$ & $6(8.21)$ & & \\
\hline \multicolumn{6}{|l|}{ Lesion character } \\
\hline & Mean \pm SD & $17.14 \pm 4.52$ & $15.00 \pm 4.29$ & 0.003 & Significant \\
\hline & Median & 17.00 & 14.00 & & \\
\hline GAGS Grade, & Mild & $48(65.75)$ & $57(78.08)$ & 0.097 & NS \\
\hline No. $(\%)$ & Moderate & $25(34.24)$ & $16(21.91)$ & & \\
\hline Non inflammatory & Mean \pm SD & $118.60 \pm 79.99$ & $124.78 \pm 116.94$ & 0.648 & NS \\
\hline lesions & Median & 106.00 & 83.00 & & \\
\hline Inflammatory & Mean \pm SD & $18.11 \pm 15.53$ & $12.47 \pm 11.36$ & 0.013 & Significant \\
\hline lesions & Median & 13.00 & 9.00 & & \\
\hline \multirow[t]{2}{*}{ Total lesions } & Mean \pm SD & $136.58 \pm 82.07$ & $137.11 \pm 118.25$ & 0.364 & NS \\
\hline & Median & 121.00 & 101.00 & & \\
\hline
\end{tabular}

Table 2: Median percent change in GAGS score and lesion count from baseline to week 12 (LOCF, ITT)

\begin{tabular}{|l|c|c|c|}
\hline Variable & Tazarotene/clindamycin & Tretinoin/clindamycin & P value \\
\hline GAGS score & $-57.14 \%$ & $-33.33 \%$ & 0.000 \\
\hline Total lesion count & $-87.17 \%$ & $-80.20 \%$ & 0.001 \\
\hline Inflammatory lesion count & $-89.15 \%$ & $-77.33 \%$ & 0.027 \\
\hline Non-inflammatory lesion count & $-85.77 \%$ & $-80.00 \%$ & 0.004 \\
\hline
\end{tabular}




\section{Original Article}

Figure 1. Flow diagram of patients
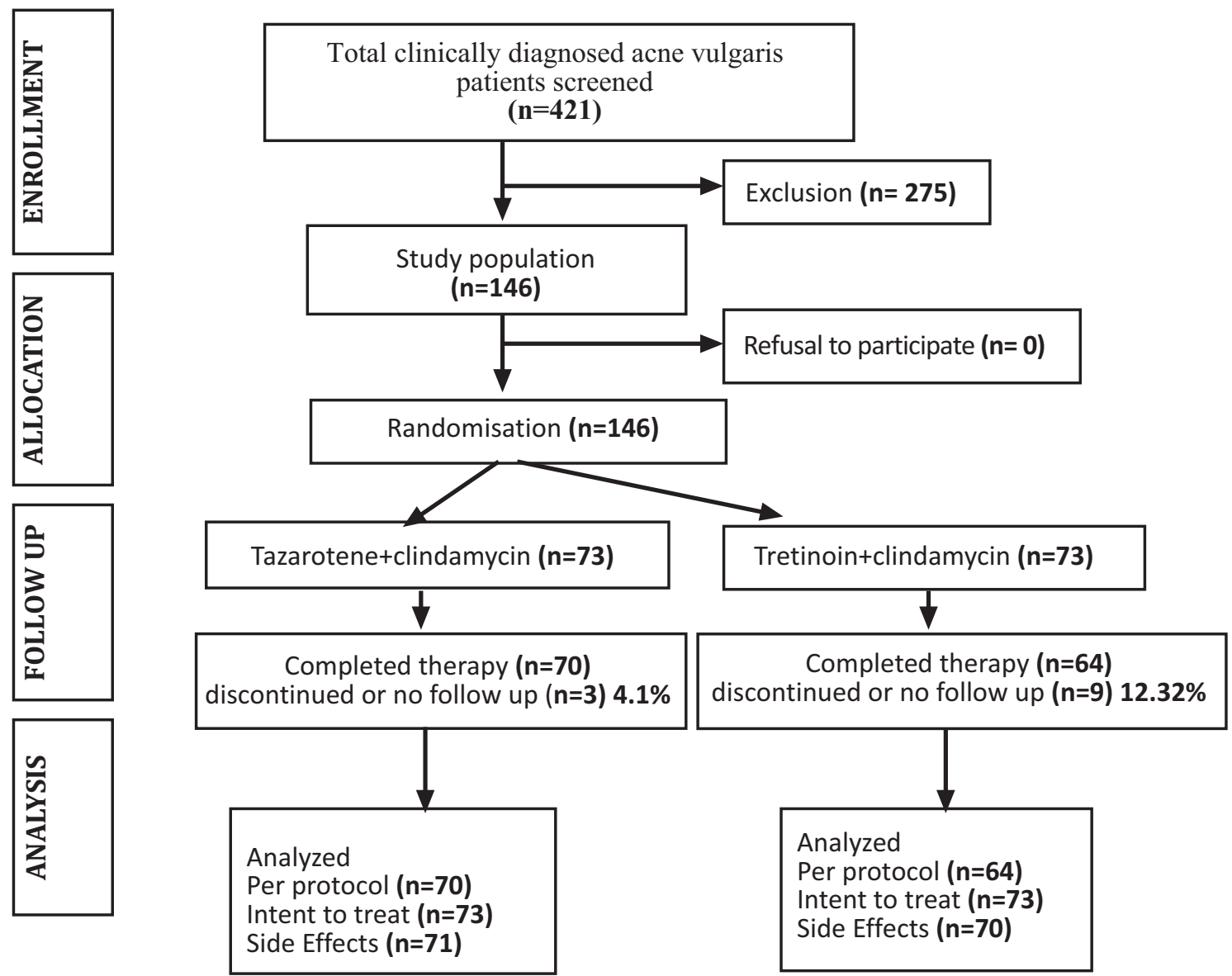

Figure 2. Percent reduction in GAGS score in both the groups.

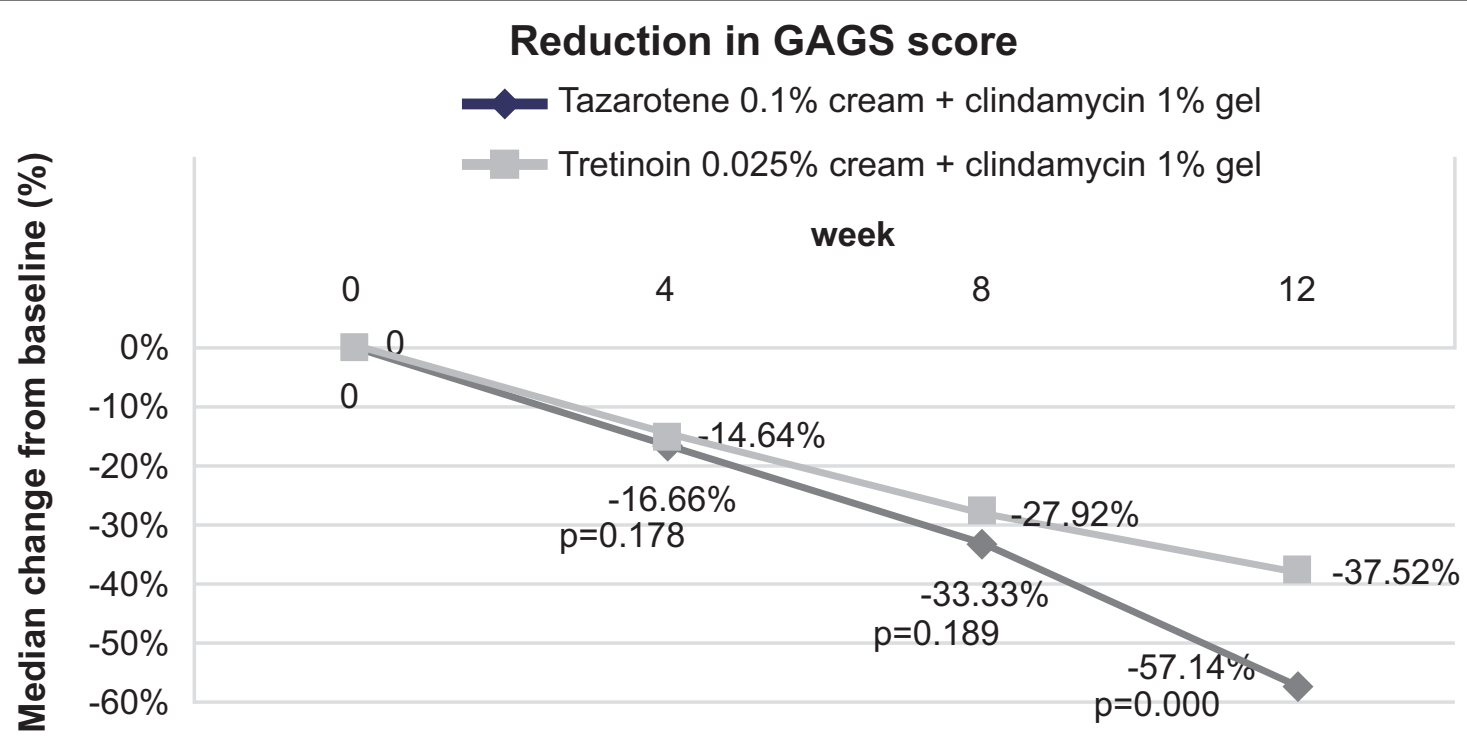




\section{Original Article}

Figure 3. Rate of reduction in non-inflammatory lesion count in both the groups

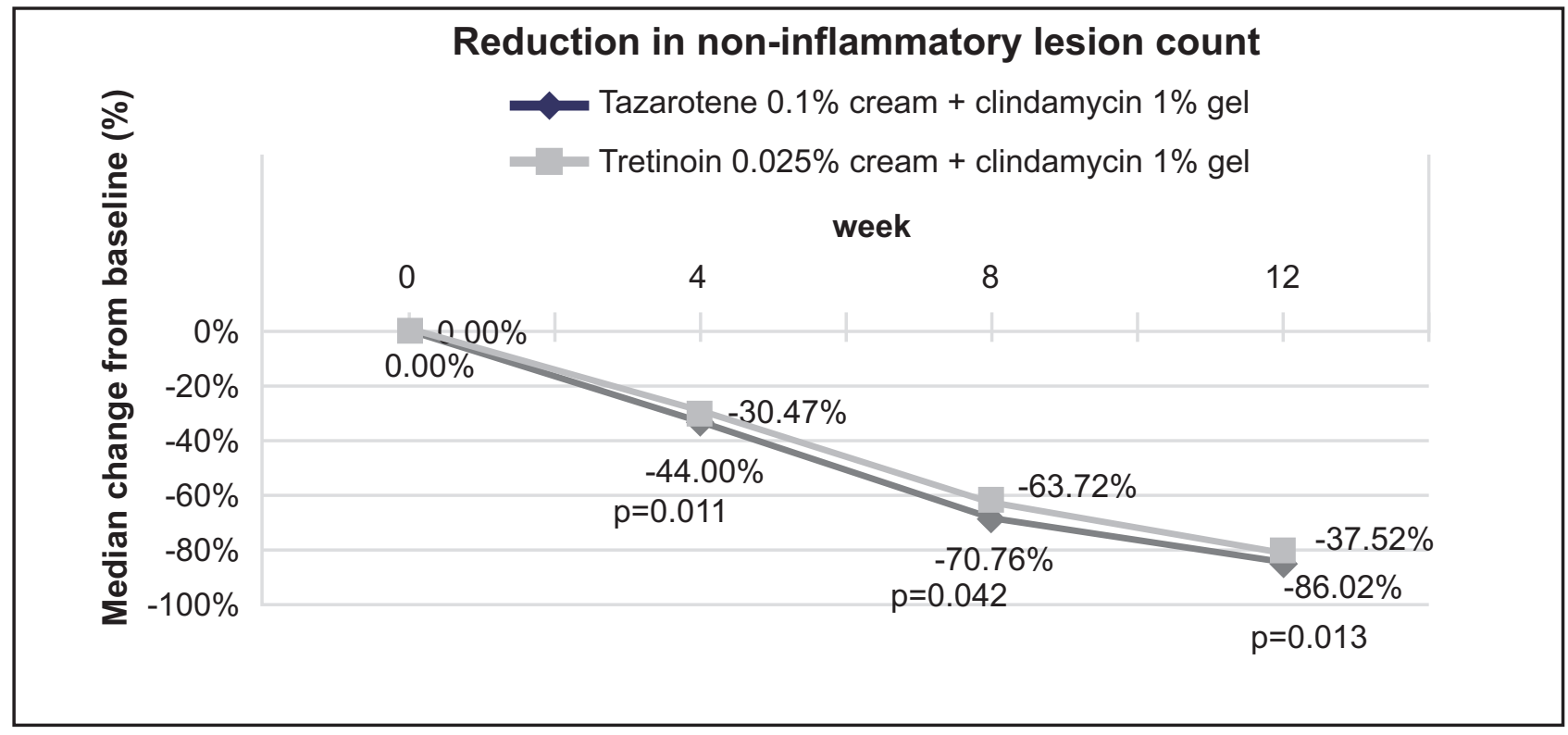

Figure 4. Rate of reduction in inflammatory lesion count in both the groups

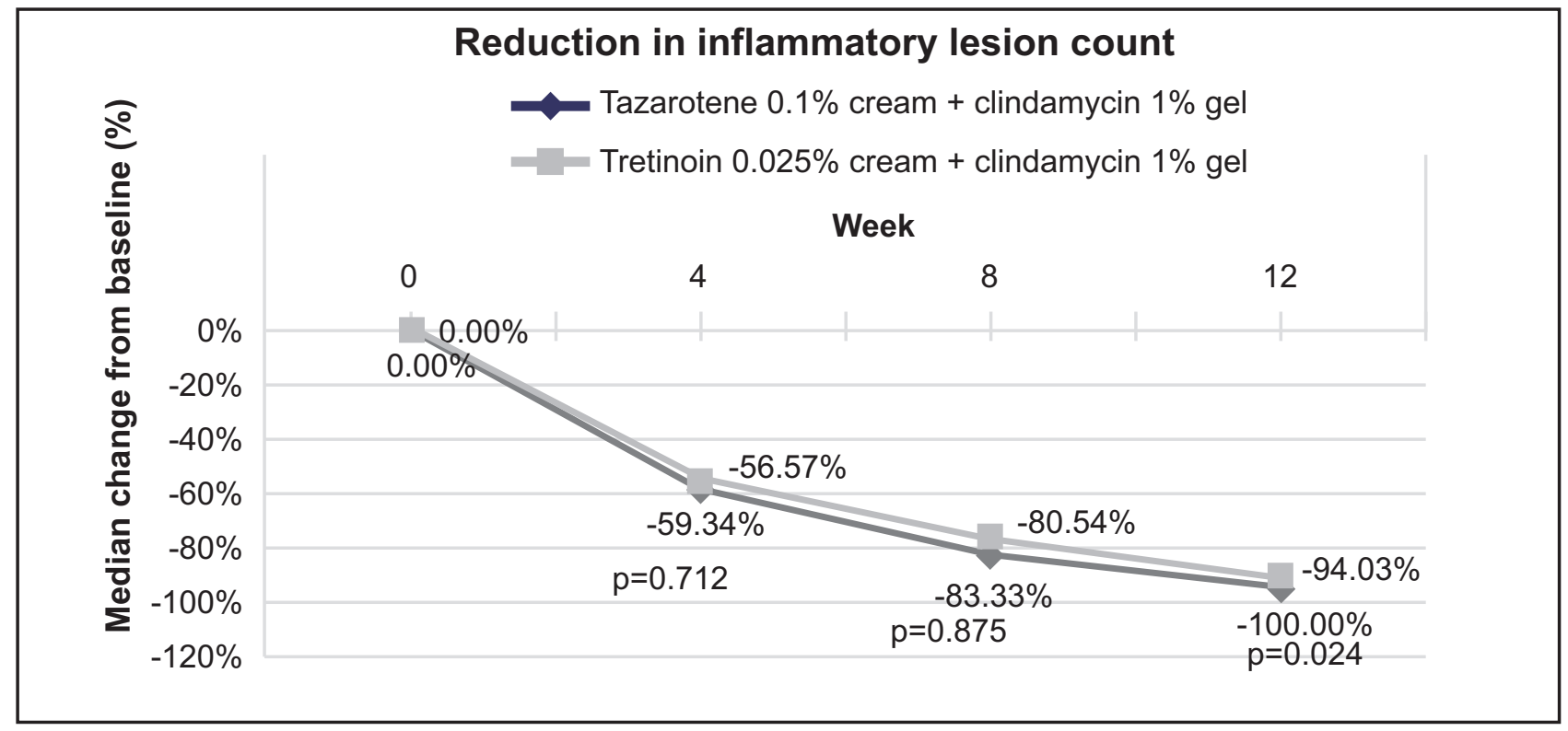




\section{Original Article}

Figure 5. Rate of reduction in total lesion count in both the groups

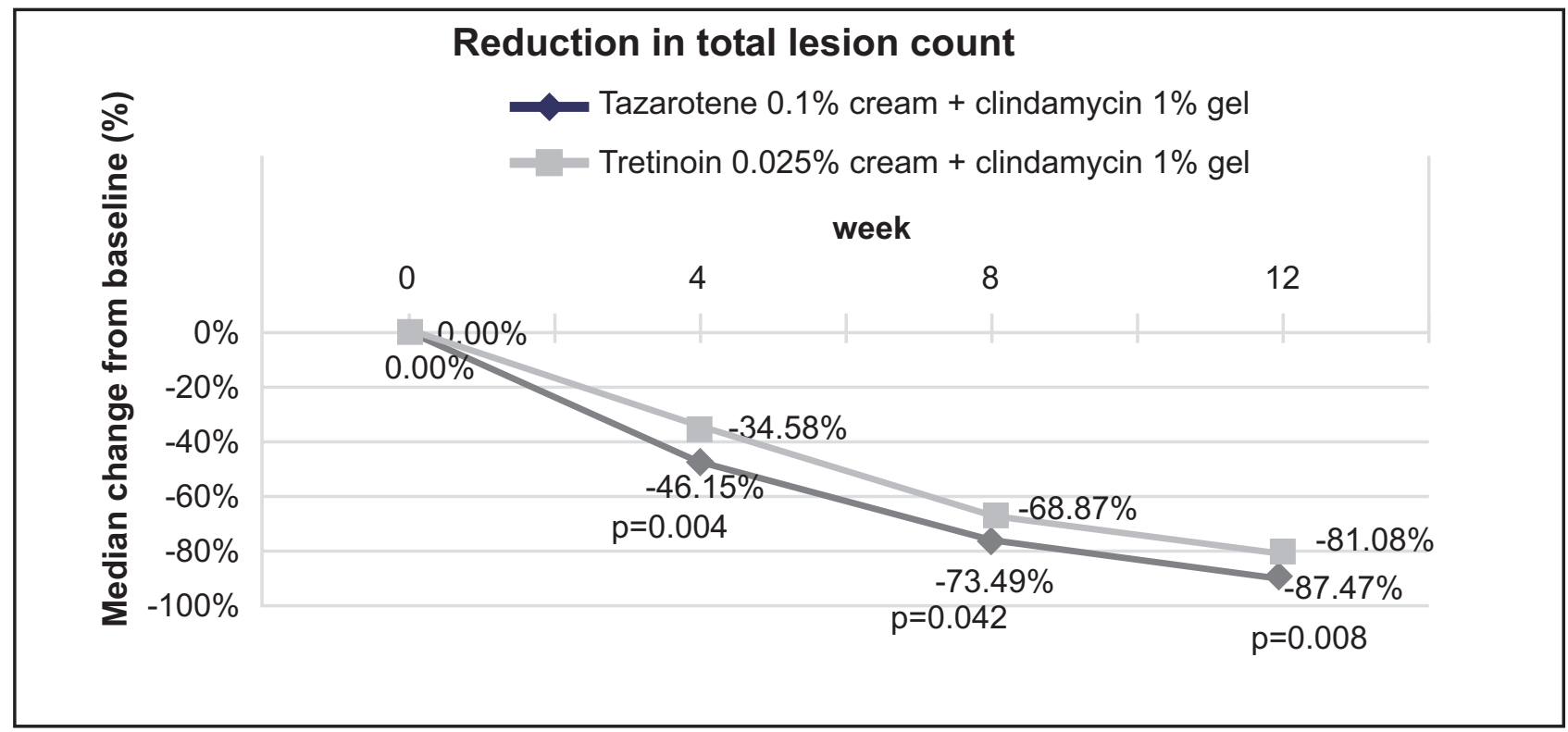

Figure 6. Percentage of the patient with at least $50 \%$ reduction in GAGS score and lesion count from baseline to 12 weeks (ITT population, LOCF)

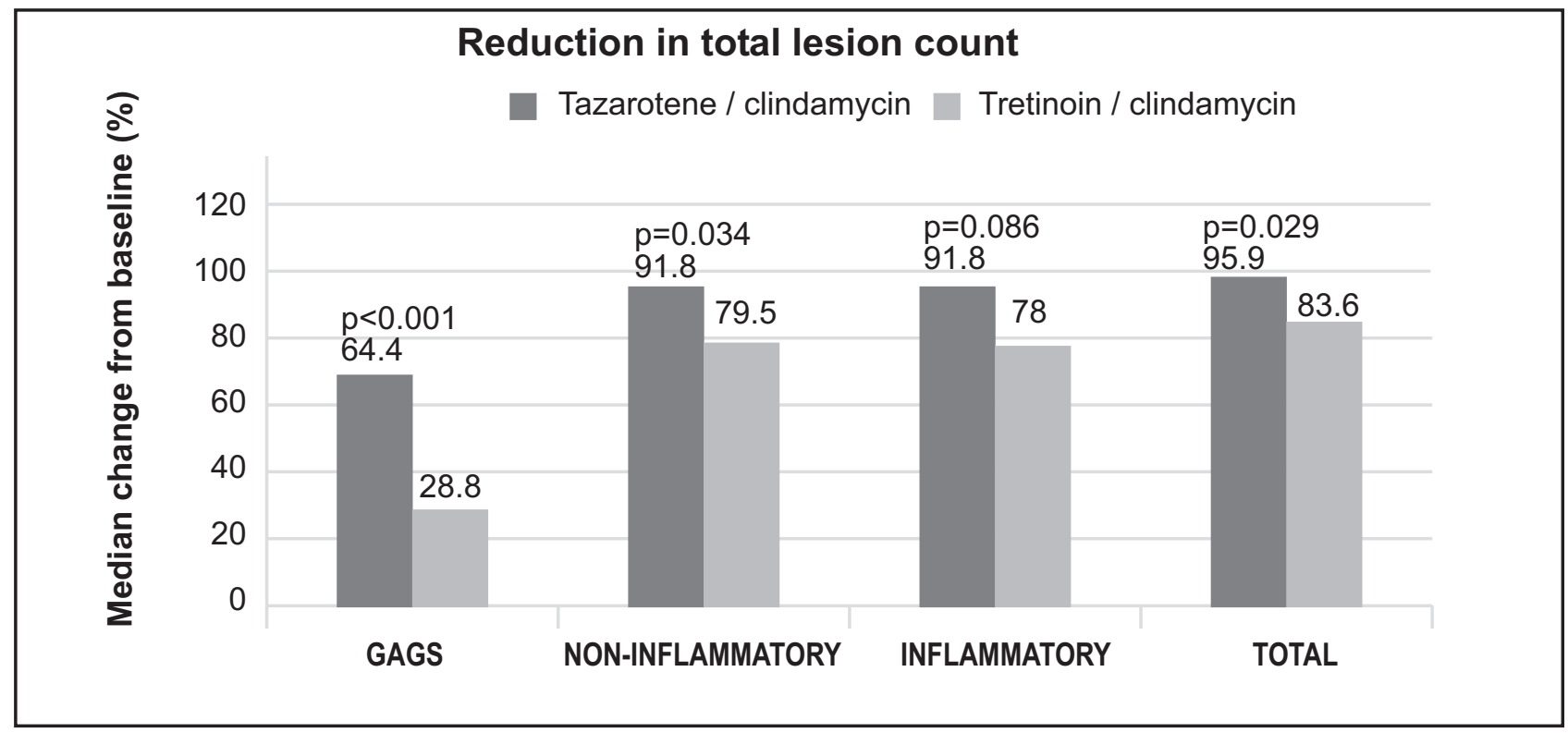


Vol. 11, No. 1, 2013

\section{Original Article}

Figure 7. Impact of acne on overall health related quality of life before and after the treatment in both the groups
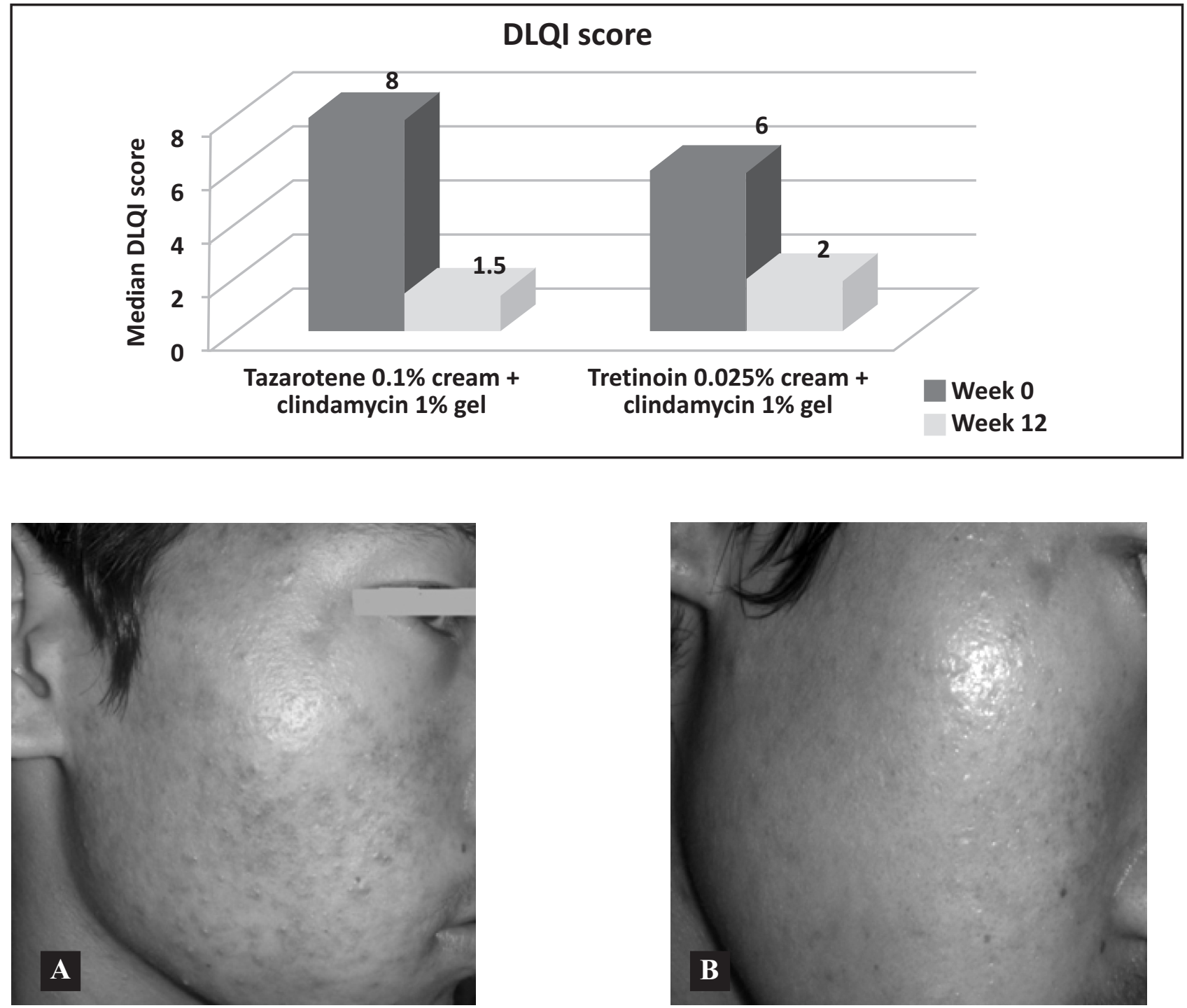

Figure 8. Tazarotene/clindamycin regimen, (A) before treatment, (B) after 12 weeks
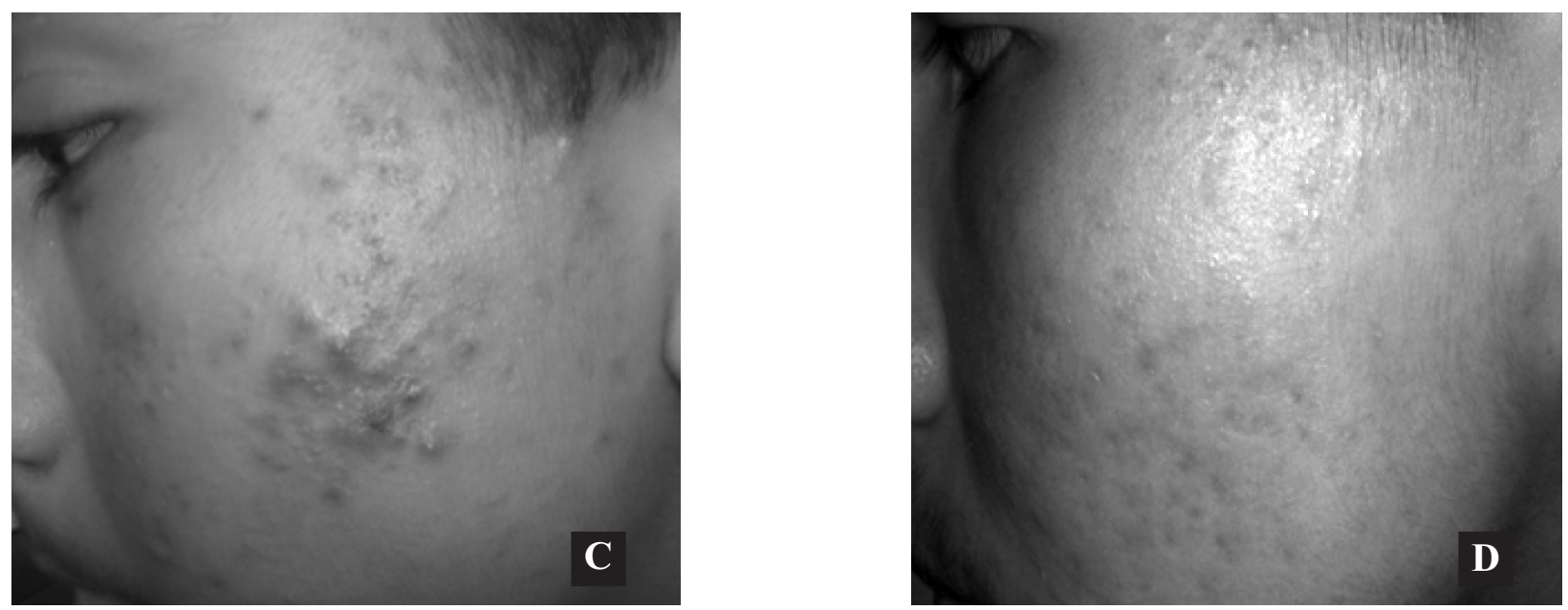

Figure 9. Tretinoin/clindamycin regimen, (C) before treatment. (D) after 12 weeks 


\section{Original Article}

\section{References:}

1. Cunliffe WJ, Gould DJ. Prevalence of facial acne vulgaris in late adolescence and in adults. BMJ 1979; 1:1109-10.

2. Gollnick H, Cunliffe W, Berson D, Dreno B, Finlay A, Leyden J J et al. Management of acne: a report from a global alliance to improve outcomes in acne. $J$ Am Acad Dermatol 2003; 49:S1-38.

3. Tanghetti E, Dhawn S, Torok H, Kircik L. Tazarotene 0.1 percent cream plus clindamycin 1 percent gel versus tretinoin 0.025 percent gel plus clindamycin 1 percent gel in the treatment of facial acne vulgaris. Dermatol Online J 2007; 13:1-9.

4. Shalita AR, Chalker DK, Griffith RF, Herbert AA, Hickman JG, Maloney JM et al. Tazarotene gel is safe and effective in acne vulgaris: a multicenter double blind randomized controlled study. Cutis 1999; 63:349-53.

5. Simpson NB, Cunliffe WJ. Disorders of Sebaceous Glands. In: Burns T, Breathnach S, Cox N, et al, editors. Rook's Textbook of Dermatology. 7th ed. Oxford: Blackwell Science; 2004. p.43.15-58.

6. Haider A, Shaw JC. Treatment of acne vulgaris. JAMA 2004; 292:726-35.

7. Webster GF, Berson D, Stein LF, Fivenson DP, Tanghetti EA, Ling M. Efficacy and tolerability of once-daily tazarotene $0.1 \%$ gel versus once-daily tretinoin $0.025 \%$ gel in the treatment of facial acne vulgaris: a randomized trial. Cutis 2001; 67:S4-9.

8. Leyden JJ, Tanghetti EA, Miller B, Ung M, Berson D, Lee J. Once-daily tazarotene $0.1 \%$ gel versus once-daily tretinoin $0.1 \%$ Microsponge gel for the treatment of facial acne vulgaris: a double-blind randomized trial. Cutis 2002; 69:S12-9.

9. Kakita L. Tazarotene versus tretinoin or adapalene in the treatment of acne vulgaris. J Am Acad Dermatol 2004; 43:S51-4.

10. Doshi A, Zaheer A, Stiller MJ. A comparison of current acne grading systems and proposal of a novel system. Int $J$ Dermatol 1997; 36:416-8.

11. Finlay AY, Khan GK. Dermatology Life Quality Index (DLQI): a simple practical measure for routine clinical use. Clin Exp Dermatol 1994; 19:210-6.

12. Korting HC, Braun-Falco O. Efficacy and tolerability of combined topical treatment of acne vulgaris with tretinoin and erythromycin in general practice. Drugs Exp Clin Res 1989; 15: 447-51.

13. Lasek RJ, Chren MM. Acne vulgaris and quality of life of adult dermatology patients. Arch Dermatol 1998; 134:454-8.

14. Baz k, Yazici K, Kokturk A, et al. Effects of isotretinoin treatment on Dermatological Quality of Life and Anxiety/ Depression in patients with Severe Acne. J Dermatol 2004;14:75-9. 